\title{
XLVIII. The origin of the prismatic colours
}

\section{Lord Rayleigh O.M. F.R.S.}

To cite this article: Lord Rayleigh O.M. F.R.S. $(1905)$ XLVIII. The origin of the prismatic colours , Philosophical Magazine Series 6, 10:58, 401-407, DOI: 10.1080/14786440509463388

To link to this article: http://dx.doi.org/10.1080/14786440509463388

册Published online: 16 Apr 2009.

Submit your article to this journal $\pi$

Џll Article views: 8

Q View related articles ¿

4 Citing articles: 1 View citing articles ๘ 
T H E

LONDON, EDINBURGH, AND DUBLIN

\title{
PHILOSOPHICAL MAGAZINE
}

\author{
AND \\ JOURNAL OF SCIENCE. \\ [SIXTH SERIES.] \\ OCTOBER 1905.
}

XLVIII. The Origin of the Prismatic Colours. By Lord Rayleigh, O.M., F.R.S.*

7 THE fact that by the aid of a spectroscope interferences may be observed with light originally white used to be regarded as a proof of the existence of periodicities in the original radiation; but it seems now to be generally agreed that these periodicities are due to the spectroscope. When a pulse strikes a grating, it is obvious that the periodicity and its variation in different directions are the work of the grating. The assertion that Newton's experiments prove the colours to be already existent in white light, is usually made in too unqualified a form.

When a prism, which has no periodicities of figure, is substituted for a grating, the modus operandi is much less obvious. This question has been especially considered by Schuster (Phil. Mag. xxxviì. p. 509, 1894 : vii. p. 1, 1904), and quite recently Ames bas given an "Elementary Discussion of the Action of a Prism upon White Light" ("Astrophysical Journal,' July 1905). The aim of the present note is merely to illustrate the matter further.

I commence by remarking that, so far as I see, there is nothing faulty or specially obscure in the traditional treatment founded upon the consideration of simple, and accordingly infinite, trains of waves. By Fourier's theorem any arbitrary disturbance may be thus compounded; and the method suffices to answer any question that may be raised, so long at least as we are content to take for granted the

* Communicated by the Author.

Plil. Mag. S. 6. Vol. 10. No. 58. Oct. 1905. 
character of the dispersive medium-the relation of velocity to wave-length-without enquiring further as to its constitution. For example, we find the resolving-power of a prism to be given by

$$
\frac{\lambda}{d \lambda}=\mathrm{T} \frac{d \mu}{d \lambda} \cdot \text {. . . . . . . }
$$

in which $\lambda$ denotes the wave-length in racuo, $\mathrm{T}$ the "thickness" of the prism, $\mu$ the refractive index, and $d \lambda$ the smallest difference of wave-length that can be resolved. A comparison with the corresponding formule for a grating shows that (1) gives the number of waves $(\lambda)$ which travel in the prescribed direction as the result of the action of the prism upon an incident pulse.

But, although reasoning on the above lines may be quite conclusive, a desire is naturally felt for a better understanding of the genesis of the sequence of waves, which seems often to be regarded as paradoxical. Probably I have been less sensible of this difficulty from my familiarity with the analogous phenomena described by Scott Russel and Kelvin, of which I have given a calculation*. "When a small obstacle, such as fishing-line, is moved forward slowly through still water, or (which, of course, comes to the same thing) is held stationary in moving water, the surface is covered with a beautiful wave-pattern, fixed relatively to the obstacle. On the up-stream side the wave-length is short, and, as Thomson has shown, the force governing the vibrations is principally cohesion. On the down-stream side the waves are longer and are governed principally by gravity. Both sets of waves move with the same velocity relatively to the water, namely, that required in order that they may maintain a fixed position relatively to the obstacle. The same condition governs the velocity, and therefore the wave-length, of those parts of the wave-pattern where the fronts are oblique to the direction of motion. If the angle between this direction and the normal to the wave-front be called $\theta$, the velocity of propagation of the waves must be equal to $v_{0} \cos \theta$, where $v_{0}$ represents the velocity of the water relatively to the (fixed) obstacle." In the laboratory the experiment may be made upon water contained in a large sponge-bath and mounted upon a revolving turn-table. The fishing-line is represented by the impact of a small jet of wind. In this phenomenon the action of a prism is somewhat closely imitated. Not only are there sequences of waves, unrepresented (as would appear)

* "The Form of Standing Waves on the Surface of Running Water," Froc. Lond. Math. Soc. xv. p. 69 (1883); Scientific Papers, ii. p. 258. 
either in the structure of the medium or in the character of the force, but the wave-length and velocity are variable according to the direction considered.

For the purposes of Scott Russel's phenomenon the localized pressure is regarded as permanent; but here it will be more instructive if we suppose it applied for a finite time only. Although the method is general, we may fix our ideas upon deep water, subject to gravity (cohesion neglected), upon which operates a pressure localized in a line and moving transversely with velocity $\mathrm{V}$. In the general two-dimensional problem thus presented, the effect of the travelling pressure is insignificant unless $\mathrm{V}$ is a possible wave-velocity; but where this condition is satisfied, a corresponding train of waves is generated. In the case of deep water under gravity the condition is always satisfied, for the wave-velocities vary from zero to infinity.

The limitation to a wave-train of velocity $\mathrm{V}$ is complete only when the time of application of the pressure is infinitely extended. Otherwise, besides the train of velocity $\mathrm{V}$ we have to deal with other trains, of velocities differing so little from $\mathrm{V}$ that during the time in question they remain sensibly in step with the first. As is known *, the behaviour of such aggregates is largely a matter of the group-velocity $\mathrm{U}$, whose value is given by

$$
\mathrm{U}=\frac{d(k \mathrm{~V})}{d k} \quad \cdot \quad \cdot \cdot \cdot \cdot \cdot
$$

$k$ being proportional to the reciprocal of the wave-length in the medium. In the particular case of deep-water waves $\mathrm{U}=\frac{1}{2} \mathrm{~V}$.

From this point of view it is easy to recognize that the total length of the train of waves generated in time $t^{\prime}$ is $\pm\left(\mathrm{V}-\mathrm{U}^{\mathrm{U}}\right) t^{\prime}$. If $\tau$ be the periodic time of these waves, the wave-length in the medium is $V \tau$, and the number of waves is therefore

$$
\pm \frac{\mathrm{V}-\mathrm{U}}{\mathrm{V}} \frac{t^{\prime}}{\mathrm{\tau}} \cdot \ldots \cdot \quad \cdot \quad \cdot
$$

But for our present purpose of establishing an analogy with prisms and their resolving-power, what we are concerned with is not the number of waves at any time in the dispersive medium itself, but rather the number after emergence of the train into a medium which is non-dispersive; and here a curious modification ensues. During the emergence the i. p. 540 .

* See, for example, 'Nature,' xxv. p. 51 (1881); 'Scientific Papers,' 
relative motion of the waves and of the group still continues, and thus we have to introduce the factor $\mathrm{V} / \mathrm{U}$, obtaining for the number $\mathrm{N}$ of waves outside

$$
\mathrm{N}=\frac{\mathrm{V}-\mathrm{U}}{\mathrm{U}} \frac{t^{\prime}}{\tau} \cdot . . . .
$$

If $\mathrm{X}$ be the distance through which the pressure travels, $\mathrm{X}=\mathrm{V} t^{\prime}$; and if $\mathrm{V}_{0}$ be the (constant) velocity outside and $\lambda$ the wave-length outside, $\lambda=V_{0} \tau$. Tbus

$$
\mathrm{N}=\left(\frac{\mathrm{V}_{0}}{\mathrm{U}}-\frac{\mathrm{V}_{0}}{\mathrm{~V}}\right) \frac{\mathrm{X}}{\lambda} \cdot \cdot \cdot \cdot \cdot
$$

To introduce optical notation, let $\beta=V_{0} / V$, so that $\mu$ is the refractive index. In terms of $\mu$

$$
\frac{V_{0}}{\mathrm{U}}=\mu-\lambda \frac{d \mu}{d \lambda} \quad \cdot \quad \cdot \quad \cdot \quad \cdot \quad \cdot
$$

so that finally

$$
\mathrm{N}=-\mathrm{X}_{d \bar{\lambda}}^{d \mu} \cdot \cdot \cdot \cdot \cdot \cdot \cdot
$$

in close correspondence with (1). A very simple formula thus expresses the number of waves (after emergence) generated by the travel of the pressure over a distance $X$ of a dispersive medium.

The above calculation has the advantage of being clear of the complication due to obliquity; but a very little modification will adapt it to the case of a prism, especially if we suppose that the waves considered are emergent at the second face of the prism without refraction. In the figure, $\mathrm{AC}$ represents an incident plane pulse whose trace runs along the first face of the prism from $A$ to $B$. $\mathrm{AF}, \mathrm{BE}$ is the direction of propagation of the refracted waves under consideration, to which the second face of the prism is perpendicular. As before, if $\tau$ be the period, $V$ the wave-velocity of the waves propagated in direction $\mathrm{BE}, \mathrm{U}$ the corresponding group-velocity, $t^{\prime}$ the time of travel of the pulse from $A$ to $B$, the number of waves within the

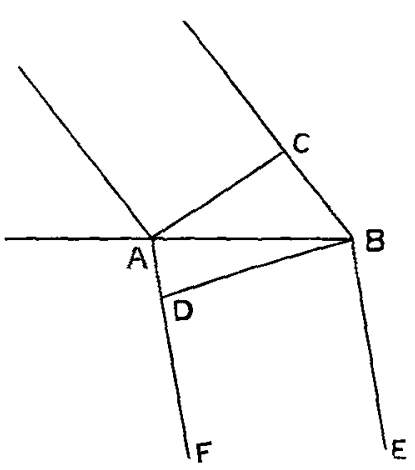
medium is

$$
\frac{\mathrm{V}-\mathrm{U}}{\mathrm{V}} \frac{\boldsymbol{t}^{\prime}}{\tau}
$$


giving on emergence the number of waves expressed in (4). If $V_{0}$ be the velocity in vacum, $\tau=\lambda / V_{0}$, and

so that

$$
t^{\prime}=\frac{\mathrm{BC}}{\mathrm{V}_{0}^{-}}=\frac{\mathrm{AD}}{\mathrm{V}^{-}}
$$

$$
\frac{t^{\prime}}{\tau}=\frac{\mathrm{AD}}{\lambda} \mathrm{V}_{0}
$$

Thus, as in (5), (6), (7),

$$
\mathrm{N}=\left(\frac{\mathrm{V}_{0}}{\overline{\mathrm{U}}}-\frac{\mathrm{V}_{0}}{\overline{\mathrm{V}}}\right) \frac{\mathrm{AD}}{\lambda}=-\operatorname{AD} \frac{d \mu}{d \lambda} . .
$$

in agreement with (1).

Although the process is less easy to follow, the construction of a train of waves from an incident pulse is as definite in the case of a prism as is that of a grating ; and its essential features are presented to the eye in Scott Russel's phenomenon.

The above treatment suffices for a general view, but it may be instructive to give an analytical statement; and this $I$ am the more inclined to do as affording an opportunity of calling attention to a rather neglected paper by Lord Kelvin entitled "On the Waves produced by a Single Impulse in Water of any Dej,th, or in a Dispersive Medium ". When we know the effect of an impulse, that of a uniform force applied for a finite time can be deduced by integration. It may be convenient to recite the leading steps of Kelvin's investigation.

Let $f(k)$ denote the velocity of propagation correspondirig to wave-length (in the medium) $2 \pi / k$. The Fourier-CauchyPoisson synthesis gives

$$
u=\int_{0}^{\infty} d k \cos k[x-t f(k)] . . \quad .
$$

for the effect at place and time $(x, t)$ of an infinitely intense disturbance at place and time $(0,0)$. When $x-t f(k)$ is very large, the parts of the integral (9) which lie on the two sides of a small range, $\kappa-\alpha$ to $\kappa+\alpha$, vanish by annulling interference ; $\boldsymbol{\kappa}$ being a value, or the value, of $k$, which makes

$$
\begin{gathered}
\frac{d}{d k}\{k[x-t f(k)]\}=0 \quad . . . \\
\text { or } \quad .
\end{gathered}
$$

* Proc. Roy. Soc. vol, xlii. p. 80 (1887). 
$\mathrm{U}$ being the group-velocity. By Taylor's theorem when $k-\kappa$ is very small,

$$
k[x-t f(k)]=\kappa^{2} t f^{\prime}(\kappa)+\frac{1}{2} t(k-\kappa)^{2}\left\{-\kappa f^{\prime \prime}(\kappa)-2 f^{\prime}(\kappa)\right\} .
$$

Using this in (9) and integrating with the aid of

$$
\int_{-\infty}^{+\infty} d \sigma \cos \sigma^{2}=\int_{-\infty}^{+\infty} d \sigma \sin \sigma^{2}=\sqrt{ }\left(\frac{1}{2} \pi\right)
$$

we find as an approximate value

$$
u=\frac{\sqrt{ }(2 \pi) \cdot \cos \left\{t \kappa^{2} f^{\prime}(\kappa)+\frac{1}{4} \pi\right\}}{\sqrt{ } t \cdot \sqrt{ }\left\{-\kappa f^{\prime \prime}(\kappa)-2 f^{\prime \prime}(\kappa)\right\}} . .
$$

As a particular case, for deep-water gravity waves

$$
\begin{gathered}
f(k)=V(g / k), \quad f^{\prime}(k)=-\frac{1}{2} g^{\frac{1}{2}} k^{-\frac{3}{2}}, \\
-k f^{\prime \prime}(k)-2 f^{\prime}(k)=\frac{1}{4} g^{\frac{1}{2}} k^{-\frac{3}{2}},
\end{gathered}
$$

and finally with use of (11)

$$
u=\pi^{\frac{1}{2}} g^{\frac{1}{2}} t x^{-\frac{3}{2}} \cos \left(\frac{g t^{2}}{4 x}-\frac{\pi}{4}\right) \text {. . . (13)*. }
$$

This gives the effect of the impulse at $(0,0)$. If the impulse be at $x^{\prime}, t^{\prime}$, we are to write $x-x^{\prime}$ for $x$ and $t-t^{\prime}$ for $t$. For our purpose of finding the effect of a travelling force, we are to make $x^{\prime}=\mathrm{V} t^{\prime}$ and integrate with respect to $t^{\prime}$ from 0 to $t^{\prime}, t^{\prime}$ being the duration of the force. The integral will depend mainly upon the part where

$$
\frac{\left(t-t^{\prime}\right)^{2}}{x-\mathrm{V} t^{\prime}}
$$

under the cosine, is stationary. This occurs when

$$
2 x=\mathrm{V}\left(t+t^{\prime}\right) \cdot \ldots \text {. . . }
$$

and then

$$
\frac{g\left(t-t^{\prime}\right)^{2}}{4\left(x-\mathrm{V} t^{\prime}\right)}=\frac{g(\mathrm{~V} t-x)}{\mathrm{V}^{2}} . . .
$$

Omitting the variation of the other factors as less important, we see that, when sensible, the effect is proportional to

$$
\cos \left\{\frac{g(\mathrm{~V} t-x)}{\mathrm{V}^{2}}-\frac{\pi}{4}\right\} \quad . \quad \cdot \text { (16), }
$$

representing simple waves of velocity $\mathrm{V}$. But this is limited to such values of $x$ and $t$ as make $t^{\prime}$ in (14) lie between 0 and $t^{\prime}$. Thus if $t$ be given, the range of $x$ is from $\frac{1}{2} \mathrm{~V} t$ to $\frac{1}{2} \mathrm{~V} t+\frac{1}{2} \mathrm{~V} t^{\prime}$; so that the train of waves covers a length $\frac{1}{2} \mathrm{~V} t^{\prime}$,

* An almost equally simple formula applies when more generally $f(k) \propto k^{n}$. 
agreeing with the general value given before, since here $\mathrm{U}=\frac{1}{2} \mathrm{~V}$. If, as would be more convenient in order to find the length of the train after emergence into a non-dispersive medium, we regard $x$ as given, we find that $t$ ranges from $2 x / \mathrm{V}$ to $2 x / \mathrm{V}+t^{\prime}$

I have taken the particular case first, as the reasoning is rather simpler when we have, as in (13), an explicit expression in terms of $x$ and $t^{*}$. In general $\kappa$ cannot be eliminated between (11) and (12), and we must proceed rather differently. The question is when will

with

$$
k[x-t f(k)] \text {. . . . . . . (17) }
$$

$$
x=t\left\{f(k)+k f^{\prime}(k)\right] \text {. }
$$

be stationary with respect to $t^{\prime}, x-\mathrm{V} t^{\prime}$ being substituted for $x$ and $t-t^{\prime}$ for $t$ in (17), (18)? Now

$$
\left(\mathrm{V} \frac{d}{d x}+\frac{d}{d t}\right)(17)=k\{\mathrm{~V}-f(k)\}+\left(\mathrm{V} \frac{d k}{d x}+\frac{d k}{d t}\right)\left(x-t \frac{d\{k f(k)\}}{d k}\right),
$$

of which the second term on the right vanishes by (18). The variation of (17) vanishes when $\mathrm{V}=f^{\prime}(k)$. Accordingly

with

$$
k\left[x-\mathrm{V} t^{\prime}-\left(t-t^{\prime}\right) f(k)\right]
$$

$$
x-\mathrm{V} t^{\prime}=\left(t-t^{\prime}\right)\left\{f(k)+k f^{\prime}(k)\right\}
$$

is stationary with respect to $t^{\prime}$, if $\mathrm{V}=f(k)$, and then assumes the form

$$
k[x-\mathrm{V} t] .
$$

Here $t^{\prime}$ must lie between 0 and $t^{\prime}$. Thus if $t$ be constant, $x$ has a range

$$
\mathrm{V} t^{\prime}-t^{\prime}\left\{f(k)+k f^{\prime}(k)\right\}=(\mathrm{V}-\mathrm{U}) t^{\prime} .
$$

And if $x$ be given, $t$ has a range

$$
t^{\prime}-\frac{V t^{\prime}}{f(k)+k f^{\prime}(k)}=\frac{t^{\prime}(\mathrm{U}-\mathrm{V})}{\mathrm{U}} \text {. . . }
$$

These are the limits over which the waves of velocity $\mathrm{V}$ extend. And (19) shows that the number of waves which pass a fixed point, either within the dispersive medium or on emergence from it, has the expression

$$
t^{\prime} \frac{\mathrm{U}-\mathrm{V}}{\mathrm{U}^{-}}
$$

where $\tau$ is the periodic time, in agreement with (4).

* For an admirable discussion of the general problem of deep-water waves arising from a localized disturbance, see Lamb, Proc. Lond. Math. Sac. vol. ii. p. 371 (1904). 